\title{
Component analysis of ECG by computer
}

\author{
DOUGLAS R. EDDY, JEAN McKENDREE, RICHARD E. McKENZIE, \\ and FREDERICK J. BREMNER \\ Trinity University, San Antonio, Texas 78284
}

\begin{abstract}
The present paper describes a set of interactive computer programs and methods (i.e., cardiac component analysis, CCA) that allow the collection, summation, and averaging of the time durations of the various ECG components. The programs are written in FORTRAN and run on a Texas Instruments minicomputer using an Intecolor 8001 terminal as the display device. The program's features include analog-to-digital conversion, color display of individual heartbeats, operator interaction, and statistical summation with tabular output. Subroutines are well structured and easily transported to other machines. Although these programs may be used on-line, it has been more efficient to perform the analyses off-line, after a subject has completed the task. Various versions of the program have been used to analyze human, baboon, and dog ECGs in 2- to 30-sec epochs, at sample rates of 256 and 1,000 samples/sec. Preliminary results from an experiment of the effects of meditation and cognitive overload are discussed.
\end{abstract}

The increasing sophistication of techniques for physiological data acquisition and the advancement of computer technology have resulted in numerous studies in search of physiological correlates of various cognitive states. Heart rate and heart rate variability or sinus arrhythmia have shown consistent changes with cognitive state and cognitive load (Coles \& Duncan-Johnson, 1975; Hyndman $\&$ Gregory, 1975). Computers have been used to identify various components of the electrocardiogram (ECG) for the purpose of detecting heart pathology in patients (Fischhof \& Larkin, 1980; Murray, Jones, \& Sanders, 1980). The present paper describes a set of interactive computer programs and methods (i.e., cardiac component analysis, CCA) that allow the collection, summation, and averaging of the time durations of the various ECG components.

The programs are written in FORTRAN and run on a Texas Instruments minicomputer using an Intecolor 8001 terminal as the display device. The computer configuration and hardware arrangement are described elsewhere (Eddy, 1979; Rodgers, 1979). The program's features include analog-to-digital (A/D) conversion, color display of individual heartbeats, operator interaction, and statistical summation with tabular output. Subroutines are well structured and easily transported to other machines. The programming algorithms used in searching through digitized ECG data for the PQRST cardiac

Requests for reprints, information, and source program listings should be addressed to Douglas R. Eddy, Department of Psychology, Trinity University, 715 Stadium Drive, San Antonio, Texas 78284. The Intecolor terminals were funded in part through a cost-sharing ISEP grant from the National Science Foundation (SER 77-10916). Programming support was funded in part through contracts from Technology Incorporated and the Air Force (F33617-78-D-0629). The authors gratefully acknowledge the early program development efforts of Barbara $A$. Boigegrain. components are similar to those of Haywood, Murphy, Harvey, and Saltzberg (1970).

\section{DESIGN CRITERIA}

The specific needs of our research dictated that the CCA program meet the following general criteria: (1) maximum flexibility for new applications, (2) option for operator intervention in correcting data acquisition "errors," and (3) statistical summary of data for PQ, QT, QR, and QRS intervals.

The criterion of maximum flexibility demanded that the programs be written in a high-level language and in a highly structured manner such that they could be easily transported to various other machines. The software had to be easily modifiable to accommodate different data sampling rates, durations of sampling, and statistical averaging options. The criterion of operator intervention was thought to be necessary to remove data from analysis that were obviously contaminated by subject movement and other transients occasionally present in the ECG. This requirement necessitated that analyses be conducted off-line on FM tape-recorded data. The experimental research designs required means and standard deviations of the durations of the various cardiac components. Other more specific criteria included digital conversion of analog signals in the range of $\pm 5 \mathrm{~V}$, minimization of $60 \cdot \mathrm{Hz}$ noise in the calculation of cardiac component intervals, display of digitized heartbeats on an Intecolor graphics terminal indicating $Q$, $R$, and $S$ wave peak values, $P$-wave initiation and $T$-wave termination, and summary statistics in tabular form for each subject.

\section{PROGRAM DESIGN}

To obtain maximum flexibility, all software was 
written in structured FORTRAN whenever possible. Subroutines were used freely to modularize functions performed within the program to obtain flexibility for future applications, new algorithms, and other modifications. The main program, labeled CCA22, and its associated subroutines are shown in Figure 1.

The indentation in Figure 1 represents the hierarchical level of each subroutine. CCA22 calls the six subroutines in the first level of indentation, provides the vectors for storing the ECG digitized data and their first derivatives, writes error messages when necessary, calculates the four component durations, and terminates the program after completing the required number of heartbeat analyses. Different applications of the CCA require that some of the first-level subroutines be modified; therefore, the descriptions that follow are in general terms only.

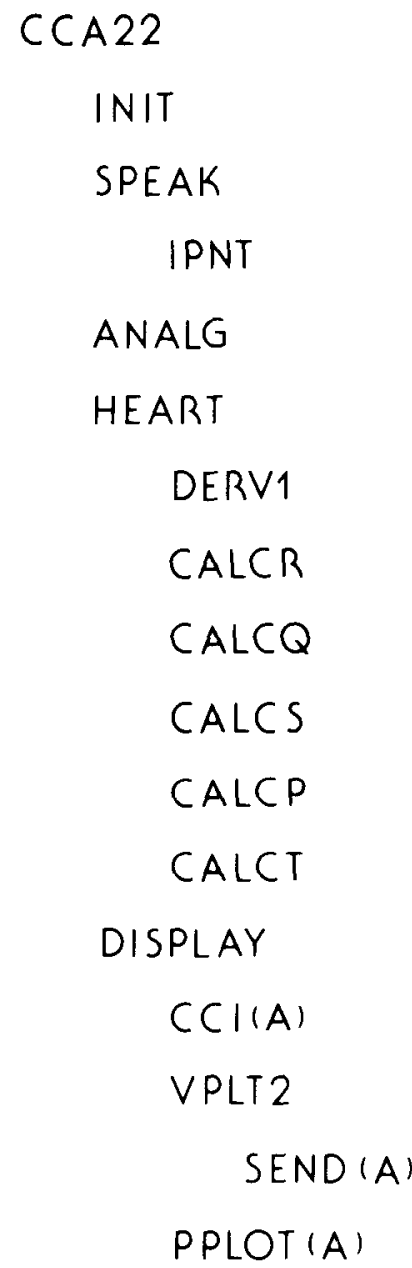

\section{SAVE2}

Figure 1. CCA22 is the main calling routine. All subroutines directly called from CCA22 are indented once. Other subroutines indirectly called are indented appropriately under the subroutine name that calls them. Subroutine names followed by a parenthesized A contain some in-line assembly language statements.
The subroutine INIT initializes several variables by requesting from the operator the analog channel to be converted, the subject number, and the terminal to be used for graphics display. The subroutine SPEAK allows the operator to control the onset of $A / D$ conversion either manually or from a code signal associated with the recorded ECG analog signal. The IPNT subroutine allows $A / D$ conversion of the code signal. Conversion of the ECG analog signal to timed digital points is performed by ANALG. If an analog value is beyond the converter's $10 . \mathrm{V}$ range, an error flag is set.

After the ECG is digitized, control returns to the main program, CCA22, which checks the error flag. An error causes the entire sample epoch to be discarded, an error message to be printed, and program control to be returned to SPEAK. Subroutine HEART is activated only if the digitized data have passed the amplitude test for within-range values. The main functions of HEART are to control the sequence of the cardiac component processors, pass the calculated maximums and minimums, and terminate further processing if a heartbeat is located too close to the beginning or ending of a sampling epoch. The latter condition eventually causes control to flow to ANALG, resulting in the digitization of a new epoch.

The first subroutine called by HEART calculates a difference vector and is called DERV1. The first derivative of the ECG is not calculated by subtracting adjacent digitized values (e.g., 1/256 sec apart), but rather, by subtracting values spaced $1 / 60 \mathrm{sec}$ apart. This technique filters the $60-\mathrm{Hz}$ noise from the difference vector (Haywood et al., 1970). For example, with 256 samples/ $\mathrm{sec}$, the sequence of differences $\mathrm{D}(\mathrm{i})$ is formed by: $\mathrm{D}(\mathrm{i})=\mathrm{V}(\mathrm{i}+2)-\mathrm{V}(\mathrm{i}-2)$, where $\mathrm{V}(\mathrm{i}+2)$ indicates the ECG voltage $2 / 256 \mathrm{sec}$ after Time Point $\mathrm{i}$ and $\mathrm{V}(\mathrm{i}-2)$ indicates a value $2 / 256 \mathrm{sec}$ before Time Point $i$. The subscript (time point) of the maximum positive difference, labeled a in Figure 2, is passed back to HEART, for later use in searching for the R-wave peak in CALCR and the Q-wave in CALCQ.

Following DERV1, subroutine CALCR receives the digitized data, the difference vector, and the subscript of the largest difference in the vector. In CALCR, the minimum difference is located between two points in the difference vector, the maximum difference (a) and $110 \mathrm{msec}$ after the maximum difference. The subscripts for the maximum and minimum differences, shown as $a$ and $b$ in Figure 2, are used as the search limits for the peak voltage of the $R$ wave. The minimum difference found in CALCR is used as the lower limit in searching for the $S$ wave in the CALCS subroutine, which is called next. The CALCQ and CALCS subroutines use the location of maxima and minima found in CALCR to begin their searches for other local maxima and minima. After establishing search limits, CALCQ and CALCS proceed as in CALCR to discover the peaks for their respective wave components. The CALCP and CALCT processors are special, in that they also search for the beginning or ending of their respective wave components. 


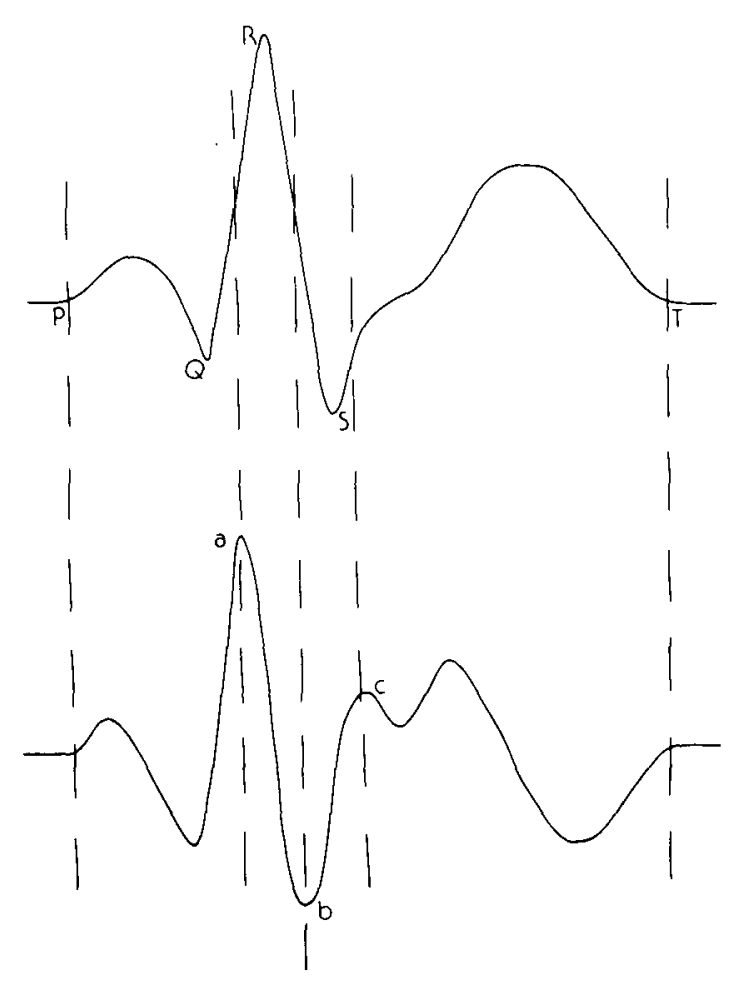

Figure 2. The upper tracing represents the ECG in its original form. The letters $Q, R$, and $S$ indicate their respective waveforms. In addition, $P$ indicates the beginning of the $P$ wave and $T$, termination of the $T$ wave. The lower tracing represents the first derivative of the original ECG. The letter a indicates the maximum positive slope, $b$, the minimum negative slope, and $c$, the maximum slope for the $T$ wave.

The CALCT processor, for example, searches the original digitized data between the maximum difference found in CALCS (c in Figure 2) and $235 \mathrm{msec}$ beyond for the peak of the $T$ component. From the subscript of the peak of $T$, the difference vector is searched for a value of zero or greater. The difference vector will contain a value of zero when the $\mathrm{T}$ component terminates (i.e., reaches a minimum). A small but important point for programming the search is to start the search through the difference vector for zero at least $20 \mathrm{msec}$ past the peak of $T$. The values of the difference vector are sometimes zero in several places near the peak, especially when fast sampling rates are used. Any local minima will cause the hill-descending algorithm to terminate its search prematurely. The beginning of the P-wave component is located in a similar manner, except that the search is performed in the reverse direction from the minimum difference obtained from CALCQ.

After each PQRST component has been identified, control returns to HEART and then to CCA22, which calls DISPLAY. The DISPLAY subroutine writes the various cardiac component intervals to the Intecolor graphics screen in front of the experimenter. Next, the ECG is graphed onto the screen starting at the beginning of the $P$ wave. With 160 pixels available across the screen, $625 \mathrm{msec}$ of ECG can be plotted when 256 samples are taken each second. The digitized ECG values displayed provide an adequate window to view a normal human heartbeat, including several milliseconds past the end of the $T$ wave. After graphing the ECG, each component for $\mathrm{P}, \mathrm{Q}, \mathrm{R}, \mathrm{S}$, and $\mathrm{T}$ is plotted in a unique color. The CCI subroutine allows the display program to set the foreground and background colors and the blink status of each character sent to the Intecolor screen. The VPLT2 subroutine allows lines to be graphed by simply specifying their endpoints. The SEND subroutine allows DISPLAY to send control codes to the graphics terminal. Subroutine PLOT allows DISPLAY to activate individual screen pixels for plotting the points used to indicate each component.

When the display is complete, control returns to CCA22, which calls SAVE2. This subroutine questions the operator as to whether or not the analysis of the displayed ECG is to be included in the summary statistics. If the ECG is free of transients and the analysis looks appropriate, the cardiac component intervals are stored and printed along with the time and date. Upon the storage of the 10th ECG, a statistical summary is produced, including the mean and standard deviation of each cardiac component interval. All sums are cleared, and new headers are printed in preparation for the next block of 10 ECGs. Regardless of whether the analysis was saved, control returns to CCA22 and then to SPEAK, at which the operator can stop or continue with further analyses.

\section{PROGRAM USE}

The most complete application of the ECG program has been in an honors thesis on electrocardiographic correlates of psychological state (McKendree, 1982). Subjects were run under a meditation exercise and a cognitive overload task in a repeated-measures design. The PQRST cardiac components were compared under each condition to ascertain which of the components, if any, might reflect a change in psychological state.

Subjects were placed in a sound-attenuated chamber in front of an Intelligent Systems Corporation Intecolor graphics terminal. The positive ECG lead was placed on the right midclavicular line over the second intercostal space. The negative electrode was on the left midclavicular line over the fifth intercostal space. A "ground" was placed over the first cervical vertebra. The electrode leads were connected to a Data Inc. differential preamplifier set at a gain of 200 with a high-frequency roll-off of $500 \mathrm{~Hz}$ and a low-frequency roll-off of $.2 \mathrm{~Hz}$. The signal was then amplified through a Beckman Type $\mathrm{T}$ encephalograph and recorded on a Vetter Series 7000 eight-channel tape recorder.

The meditation exercise involved visual imaging by the subject of a favorite relaxation situation, with his eyes open. The cognitive overload task, originally described by Perelli (1980), involved memorization of a list of five digits and their associated symbols before the testing session. An array of digits and one symbol 
were presented to the subject, who had to press a button corresponding to the correct digit associated with the symbol. The computer-controlled displays were presented at successively shorter delays until the subject could no longer respond. The two conditions were presented to the subject three times, with meditation always followed by the cognitive task.

The CCA22 program was used to sample and analyze the recorded data. Two seconds of data were digitized taking 256 points/sec. The program found the largest positive slope in the digitized data to determine which heartbeat to analyze. Data sampling was initiated by pressing the return key on the operator's terminal. The revolution counter of the recorder and the recorded code signal from the tape were used to locate the appropriate data for sampling. The ECG data were analyzed off-line.

Statistical analyses were performed on the mean cardiac component intervals, resulting in statistical significance for the PQ and ST components. Both components were consistently shortened in duration during the cognitive task and lengthened under the meditation condition.

\section{EVALUATION}

McKendree (1982) found the CCA22 program to be accurate and reliable. Of the heartbeats analyzed, approximately $7 \%$ had to be discarded from further analysis. Causes for rejected heartbeats were lack of a distinct $P$ wave because of electrode placement and the occurrence of muscle potentials. During relaxational sequences when movement was minimal, very few analyses had to be rejected. Tape-recorder head misalignment caused a low-frequency artifact that occasionally distorted the ECG wave forms until the problem was corrected.

Other versions of the program analyze the ECGs of acute dog preparations in a mammalian physiology course and the ECGs of baboons riding a centrifuge. The dog ECG program was very similar to the version McKendree (1982) used with humans; however, several hardware problems prevented completion of the work. The baboon ECG program was extensively modified along several dimensions. The program was moved to an IBM 3031 computer. The digitized points were read from a digital tape produced by another computer. requiring ANALG to be replaced by a read statement. The sample points were digitized at 1,000 points/sec on each of three channels. All heartbeats were analyzed within a 5-sec epoch, requiring a complicated search algorithm. In addition, subroutines for moving the computer's selected $P, Q, R, S$, and $T$ points to other locations on the ECG were added.

The baboon ECGs were highly contaminated with $60-\mathrm{Hz}$ noise and extensive chest muscle artifact. The programs performed reasonably well but required operator intervention on approximately $30 \%$ of the heartbeats. Because the graphics terminals on the IBM machine could not be run faster than 1,200 baud at the time of testing, slow interactive graphics were unacceptable to most operators. Running this type of analysis on a large mainframe is very expensive, approximately $\$ 11.50$ for 5,000 time points (15-18 heartbeats).

These programs should be an asset to anyone interested in the objective measurement of the various components of the ECG.

\section{REFERENCES}

Coles, M. G., \& Duncan-Johnson, C. C. Cardiac activity and information processing: The effects of stimulus significance and detection and response requirements. Journal of Experimental Psychology, 1975, 1, 418-428.

EdDy, D. R. Application of color displays to problems in the undergraduate laboratory. Behavior Research Methods \& Instrumentation, 1979, 11, 131-134.

Fischhof, T. J., \& Larkin, H. Computer measurement of electrocardiograms: A study of left ventricular hypertrophy in borderline hypertensive young men. International Journal of Biomedical Computing, 1980, 11, 379-387.

Haywood, L. J., Murphy, V. K., Harvey, G. A., \& Saltzberg, S. On-line real time computer algorithm for monitoring the ECG waveform. Computers and Biomedical Research, 1970, 3, 15-25.

Hyndman, B. W., \& Gregory, J. R. Spectral analysis of sinus arrhythmia during mental loading. Ergonomics, 1975, 18, 255-260.

McKendRE E, J. E. Electrocardiographic correlates of psychological state. Texas Psychologist, 1982, 34, 13-16.

Murray, T. M., Jones, W. T., \& Sanders, C. A. A real-time microprocessor-based ECG contour analysis system utilizing decision tables. IEEE Transactions in Biomedical Engineering, 1980, 27, 358-363.

Perelli, L. Effects of fatigue stressors on flying performance. Unpublished doctoral dissertation, Catholic University of America, 1980.

Rodgens, F. Microprocessor software applications in color graphics. Behavior Research Methods \& Instrumentation, 1979, 11, 135-138. 